Q

Check for

Cipdates

Cite as

Nano-Micro Lett.

(2020) 12:35

Received: 19 October 2019

Accepted: 14 December 2019

Published online: 22 January 2020

(C) The Author(s) 2020

\section{Electrical Excitation of Long-Range Surface Plasmons in PC/OLED Structure with Two Metal Nanolayers}

\author{
Valery Konopsky ${ }^{1}$, Valery Prokhorov ${ }^{2}$, Dmitry Lypenko² ${ }^{2}$, Artem Dmitriev², \\ Elena Alieva ${ }^{1}$, Giovanni Dietler ${ }^{3}$, Sergey Sekatskii ${ }^{3}$ \\ $\triangle$ Valery Konopsky, konopsky@gmail.com \\ 1 Institute of Spectroscopy, Russian Academy of Sciences, Fizicheskaya, 5, Troitsk, Moscow, Russia 108840 \\ 2 Frumkin Institute of Physical Chemistry and Electrochemistry Russian Academy of Sciences, Leninsky pr., \\ 31/4, Moscow, Russia 119071 \\ 3 Laboratoire de Physique de La Matière Vivante, IPHYS, Ecole Polytechnique Fédérale de Lausanne, \\ 1015 Lausanne, Switzerland
}

\title{
HIGHLIGHTS
}

- Long-range surface plasmons were first excited in a hybrid photonic-crystal/organic-light-emitting-diode microstructure containing two metal nanolayers.

- These surface plasmons were excited without any external laser light, but by injecting current through the two metal nanolayers, which serve as thin metal electrodes for organic light-emitting microfilm between the layers.

ABSTRACT A current-driven source of long-range surface plasmons (LRSPs) on a duplex metal nanolayer is reported. Electrical excitation of LRSPs was experimentally observed in a planar structure, where an organic light-emitting film was sandwiched between two metal nanolayers that served as electrodes. To achieve the LRSP propagation in these metal nanolayers at the interface with air, the light-emitting structure was bordered by a one-dimensional photonic crystal (PC) on the other side. The dispersion of the light emitted by such a hybrid PC/organic-light-emitting-diode structure (PC/OLED) comprising two thin metal electrodes was obtained, with a clearly identified LRSP resonance peak.

KEYWORDS Surface plasmons; Photonic crystal waveguides; Light-emitting polymers

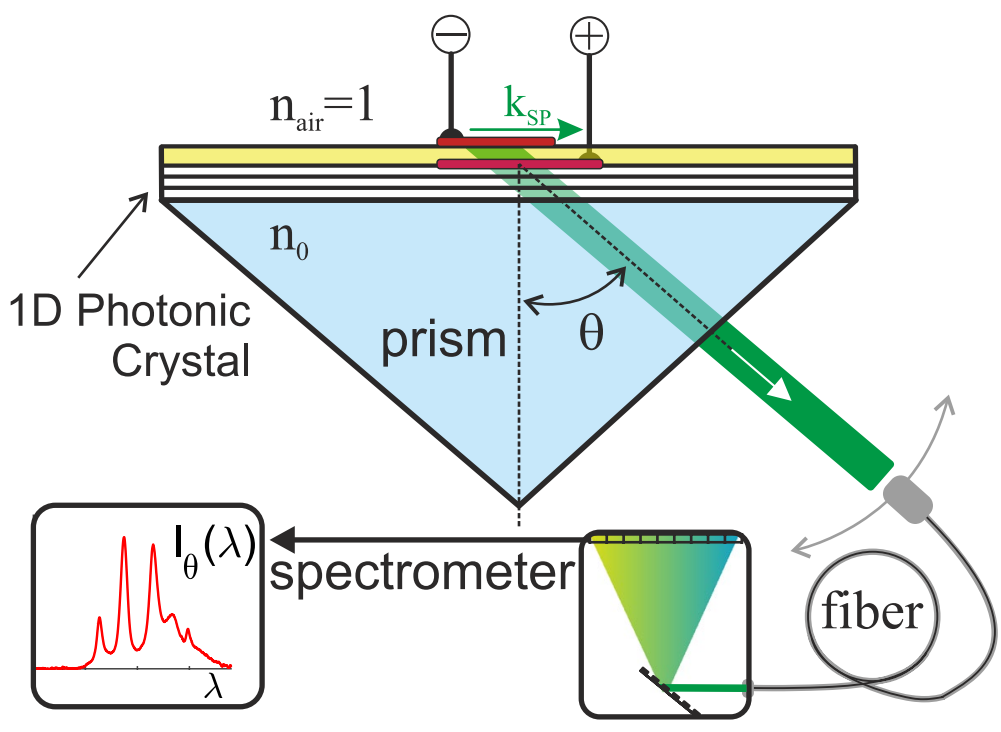




\section{Introduction}

The broad interest in the problems associated with surface plasmon (SP) amplification and lasing, witnessed for at least 20 years, has been driven by the practical necessities of the miniaturization of electronic and optical devices: lowering power consumption, increasing the operating frequency range, requiring increasing degrees of integration. There is a general belief that optics-based approaches, due to their inherent advantages over electronic ones, will progressively replace the latter. The twenty-first century has often been referred to as the "century of photons" in the same sense that the previous century was that of electrons. Profound scientific studies and technological research in the field of surface plasmon lasing, starting with the SPASER proposal in 2003 [1] and the subsequent experimental realization of such, or similar, devices [2-10], are essential steps in this direction.

However, without the denial of substantial and real progress, two requirements (which, as of today, have been seldom met) should be emphasized. First, any surface source of light that can work in the field of future photonics or its interface with electronics must not depend on an external bulk laser (for corresponding operational purposes) providing optical pumping. Second, the generation of surface plasmons propagating exactly along the corresponding interface(s), rather than, for example, lasing in the direction perpendicular to the surface with the nanoplasmonic particles/structures, is needed.
In this work our first results with regard to the fabrication of such devices are reported. In the system under study, a current injection-driven organic light-emitting diode (OLED) is sandwiched between two thin metal layers (Au and $\mathrm{Al}$ electrodes) and deposited on a 1D photonic crystal (PC). Thus, the complete multilayer structure, shown in detail in Fig. 1, comprises the following layers: [PC/ $\left.\mathrm{M}_{1} / \mathrm{OLED} / \mathrm{M}_{2} / \mathrm{air}\right]$. The integral thickness of the OLED is selected such that both metal electrodes, if thin enough, can support the long-range propagation of surface plasmons.

The ability of a single metal nanolayer to support LRSP propagation in a symmetric dielectric/thin-metal-layer/dielectric [D/M/D] structure has been well known since the early 1980s [11, 12] and is widely used in plasmonics [13]. A multilayer structure in the form [PC/M/air] has been proposed to simplify the implementation of LRSP in practical applications, where the external dielectric is air [14]. This approach was tested with diverse systems, including thin palladium layers (for ultrasensitive hydrogen detection [15-17]), thin gold layers in blue spectral range (for nitrogen dioxide detection [18]), and thin ferromagnetic cobalt layers (for magnetoplasmonics [19]), among others (see also [20-23] for examples of other applications).

However, in the works mentioned above, structures comprising only one thin metal layer were exploited. Recently, it has been recognized that two thin metal layers, with appropriate dielectric spacing between them, also can quite effectively support long-range surface plasmon (LRSP) (a)

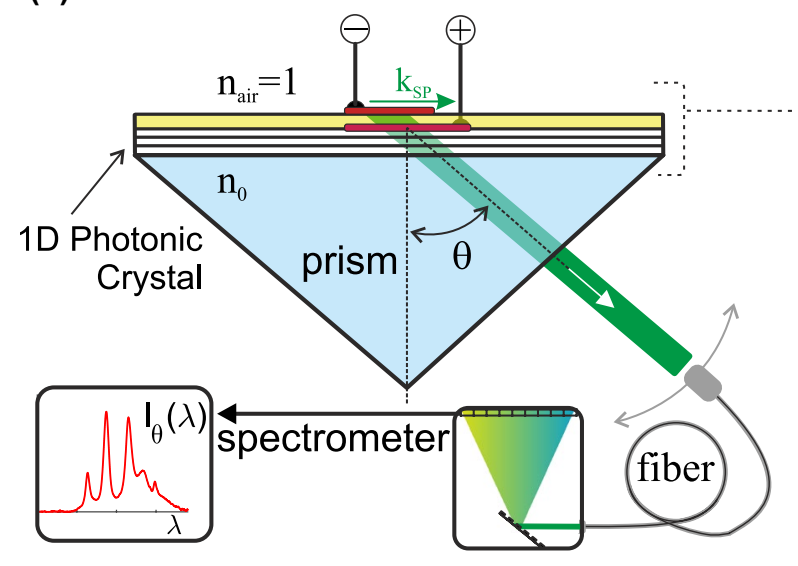

(b)

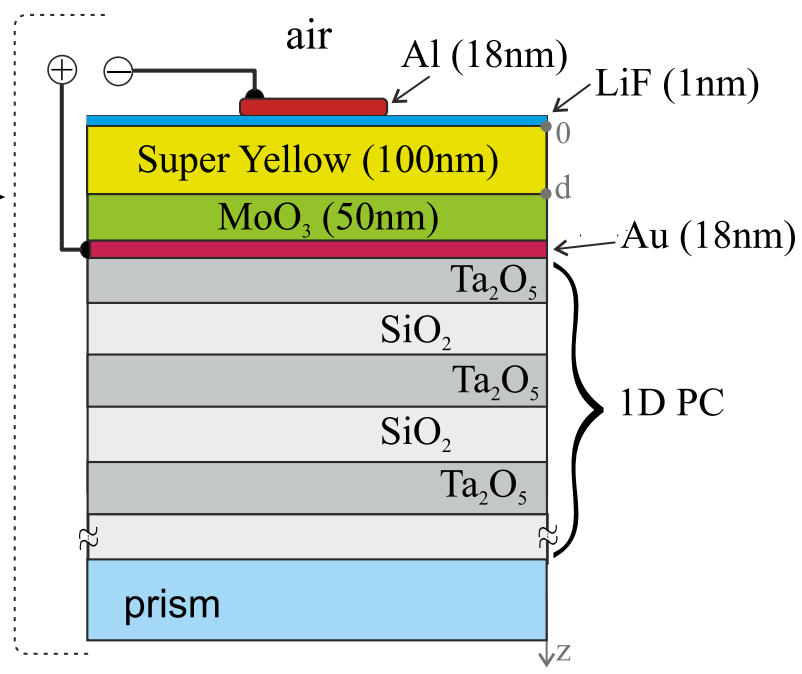

Fig. 1 a Layout of the experiment and $\mathbf{b}$ the PC/OLED structure with two metal nanolayers 
propagation [24]. This design has implemented in the present study and the current injection in the OLED is caused by the (DC) voltage applied between these two (possibly thin) metal electrodes.

\section{Plasmons in Duplex Metal Layer}

\subsection{Experimental Setup}

The experimental setup is presented in Fig. 1a. The structure in the study [PC/Au/OLED/Al/air] was placed onto the quartz right-angle prism's hypotenuse. A thin layer of immersion oil was used to attain optical continuity and refractive index (RI) matching between the substrate of the PC chip and the prism. The light emitted from the multilayer structure was collected via a multimode optical fiber with the input face rigidly attached to the rotating arm of the homemade screw-gear setup, allowing angular scanning with an accuracy of $0.25^{\circ}$. At each fixed registration angle, the spectrum of the collected light was recorded using the AvaSpec 2048 fiber-optic spectrophotometer (Netherlands), with a spectral resolution $0.04 \mathrm{~nm}$.

\subsection{D Photonic Crystal}

The 1D PC part of the multilayer structure was deposited by magnetron sputtering and has the form $[\mathrm{PC}]=[\mathrm{sub}-$ strate $\left./(H \cdot L)^{N} / H^{\prime}\right]$, where $L$ is a $\mathrm{SiO}_{2}$ layer with thickness $d_{1}=118.6 \mathrm{~nm}, H$ is a $\mathrm{Ta}_{2} \mathrm{O}_{5}$ layer with $d_{2}=86.8 \mathrm{~nm}$, and $H^{\prime}$ is a $\mathrm{Ta}_{2} \mathrm{O}_{5}$ layer with $d_{3}=75.8 \mathrm{~nm}$. The prism and the substrate were made from fused silica. The RI of the $\mathrm{Ta}_{2} \mathrm{O}_{5}$ layers is $n_{2}=n_{3}=2.11$, whereas the RIs of the substrate and prism and $\mathrm{SiO}_{2}$ layers are $n_{0}=n_{1}=1.46$ (at wavelength $\lambda=575 \mathrm{~nm}$ ). When $N=13$ (in the present case), this $\mathrm{SiO}_{2} /$ $\mathrm{Ta}_{2} \mathrm{O}_{5}$ 27-layer structure (started and finished with $\mathrm{Ta}_{2} \mathrm{O}_{5}$ layers) permits guided waves, propagating along the outer layers, to be decoupled through a prism at a resonance angle $\theta$, as shown in Fig. 1a. Thus, this scheme can be called an inverted Kretschmann geometry. If the number $N$ increases, this decoupling pathway vanishes, and only emissions through the edges of this multilayer structure are possible.

\subsection{OLED Composition}

The OLED portion of the multilayer structure has the form: $[\mathrm{OLED}]=\left[\mathrm{MoO}_{3} / \mathrm{SY} / \mathrm{LiF}\right]$, with the thicknesses of the layers shown in Fig. 1b. The RIs of the OLED layers, at $\lambda=575 \mathrm{~nm}$, are 2.0 for the transport layer $\left(\mathrm{MoO}_{3}\right), 1.86$ for the light-emitting layer (SY), and 1.39 for the barrier layer $(\mathrm{LiF})$. The $\mathrm{RIs}$ of $\mathrm{Au}$ and $\mathrm{Al}$ at this wavelength are $n_{\mathrm{Au}}=0.3+2.8 i$ and $n_{\mathrm{Al}}=1.1+6.9 i$, respectively [25-27]. The maximum brightness of a test OLED structure with the parameters mentioned above, but without the 1D PC section [glass/Au/OLED/Al/air], measured in the direction perpendicular to the layers, was $13,500 \mathrm{~cd} \mathrm{~m}^{-2}$ at $12 \mathrm{~V}$.

Super Yellow (SY, PDY-132, Merck; $M_{w}>1,300,000$, $\left.M_{n}>200,000\right)$ is an efficient electroluminescent material having a broad luminescence band centered around $570 \mathrm{~nm}$; its emission spectrum is presented in Fig. 2a as a cyan line. This material is widely used as a polymer light-emitting

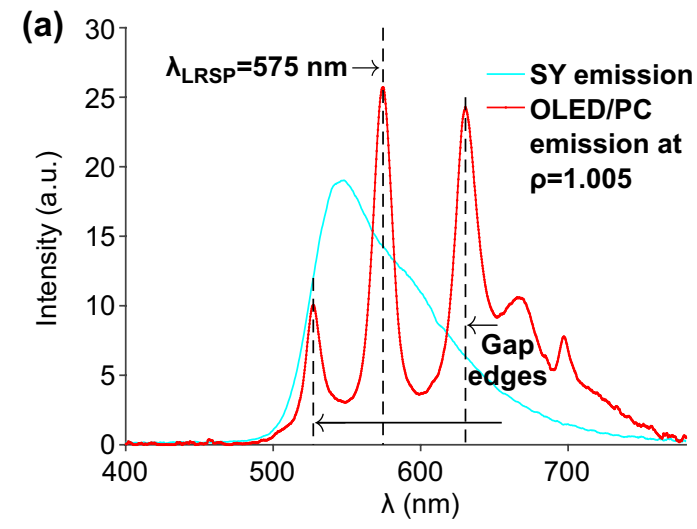

(b)

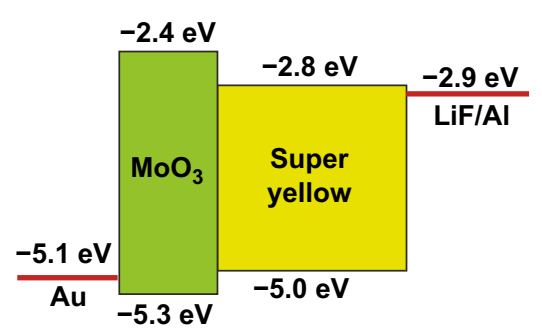

Fig. 2 a Electroluminescence spectrum from the 1D PC with the Super Yellow light-emitting layer, which is sandwiched between two metal nanolayers. The standard Super Yellow emission spectrum is presented as a cyan curve for comparison. b The energy level diagram of the OLED layers. (Color figure online) 
layer for OLED manufacturing due to its high stability and excellent brightness characteristics [28, 29]. The technical reason for choosing $\mathrm{MoO}_{3}$ (Lumtec, Taiwan) as the material for the hole injection-transport layer (HTL) is that it does not dissolve in chlorobenzene that is used for the structure preparation, namely during the spin-coating deposition of the SY layer slightly above the transport layer. Besides this technical reason, $\mathrm{MoO}_{3}$ has quite suitable alignment of energy levels for the band structure of the active layer and Au anode [30]. The energy level diagram of the OLED layers is presented in Fig. 2b. The position of the HTL energy levels is critical for OLED functioning, making it possible to reduce the drive voltage by enhancing the charge injection at the interface, thereby improving the power efficiency of the device.

\subsection{OLED Fabrication on the 1D PC}

The OLED manufacturing process was as follows. First, the surface of the 1D PC structure was irradiated by an ultraviolet lamp for 15-20 min to clean and activate the external PC surface. Next, the auxiliary 90-nm-thick layer of Al was deposited at a rate of $0.02-0.04 \mathrm{~nm} \mathrm{~s}^{-1}$ through the specially designed mask onto a part of the PC surface by thermal evaporation in vacuum at a pressure of $6 \times 10^{-6}$ Tor. This thick metal layer surrounding the OLED area was used for the subsequent attachment of external electrical contacts (electrodes). Then, the 18-nm-thick gold layer (OLED anode) was deposited through another mask by thermal evaporation using the same vacuum and rate conditions. This layer, at certain places predefined by the mask design, physically and electrically contacts the previously deposited thick Al layer and, therefore, via this layer, also with the external electrodes. Four different independent OLED structures with sizes of approximately $4 \times 4 \mathrm{~mm}^{2}$ were prepared on each $1 D$ PC chip with a diameter of $25.4 \mathrm{~mm}$.

Furthermore, a 50-nm-thick $\mathrm{MoO}_{3}$ transport layer was deposited with a rate of $0.03 \mathrm{~nm} \mathrm{~s}^{-1}$ in the same vacuum conditions. The next step was the spin-coating deposition of the SY layer over the $\mathrm{MoO}_{3}$ layer. Then, $80-90 \mu \mathrm{L}$ of a well-agitated solution of SY in chlorobenzene, with a concentration of $5 \mathrm{mg} \mathrm{mL}^{-1}$, was used for the spin coating at a rotation speed of $1000 \mathrm{rpm}$ and approximately 1 -min exposition time. Then, the sample was allowed to dry for $12 \mathrm{~h}$ at room temperature and for $4 \mathrm{~h}$ at $800{ }^{\circ} \mathrm{C}$ to remove the residual chlorobenzene traces. The OLED samples were prepared, and their spectral and photoelectric characteristics were measured at room temperature in a glove box (MBraun, Germany) under an argon atmosphere with a controlled content of oxygen and water (below $1 \mathrm{ppm}$ ). Finally, 1-nm-thick LiF and 18-nm-thick Al (cathode) layers were thermally deposited, again in the same vacuum and rate conditions as described above, with the use of the third mask. Voltage-current and voltage-brightness characteristics were measured with a Keithley 2601 SourceMeter, Keithley (USA) 485 pico-ammeter and TKA-04/3 luxmeterbrightness meter (Russia). The thicknesses of the films were determined using an MII-4 interferometer (LOMO, Saint Petersburg, Russia).

\section{Results and Discussion}

\subsection{LRSP Resonance Observation}

The red curve in Fig. 2a shows a representative example of the recorded spectra at registration angle $\rho=n_{0} \sin (\theta)=1.005$. The LRSP resonance is seen at $\lambda_{\text {LRSP }}=575 \mathrm{~nm}$, while two local maxima at 527 and $630 \mathrm{~nm}$ correspond to the bandgap edges of the structure under study. Such measurements were repeated point-by-point across the angular parameters $\varrho=0.89 \ldots 1.12$. Due to the finite sizes of the light source and the optical fiber input aperture, the recorded spectra roughly correspond to a $2^{\circ}$ convolution around the selected angle.

The stacked set of such angular measurements taken at different $\varrho$ values provides a two-dimensional picture $I(\varrho, \lambda)$, shown in Fig. 3b, which reveals a local maximum at the point $(1.003,575 \mathrm{~nm})$, corresponding to the LRSP excitation. It was previously demonstrated $[14,31]$ that the excitation of optical surface waves with an effective RI near

$\rho_{1 / 2}=n_{e}+\frac{n_{\mathrm{e}}^{3}}{2}\left[\pi \frac{d_{\mathrm{m}}}{\lambda}\right]^{2}$

is an essential prerequisite for the LRSP propagation along a thin metal film. For the classical LRSP structure [D/M/D], this condition is satisfied automatically $[31,32]$. In theoretical work [24], it was found that the same prerequisite holds for structures containing two metal nanolayers. In this case, the tangential component of the electric field is zero at the 

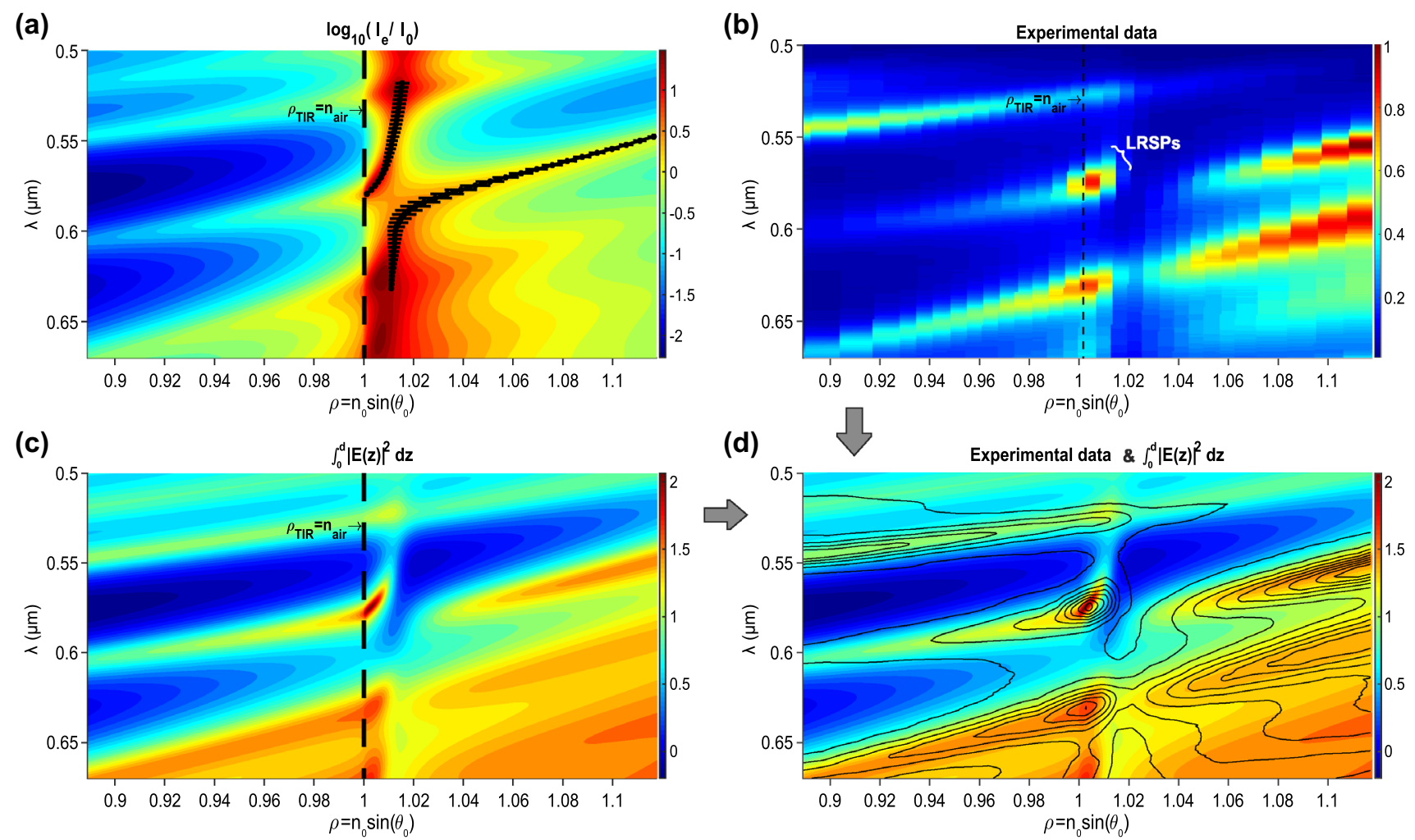

Fig. 3 Dispersion plots of the light-emitting structure under study: a calculated intensity enhancement at the external surface (in a logarithmic color scale), and the dispersion curves of surface modes for a semi-infinite 1D PC (black lines, the error bar represents the imaginary part of $\varrho$ ), b the stack of 29 experimental spectra of electroluminescence taken at different angular parameters $Q$, c calculated integral of the optical electric field in the Super Yellow layer, and $\mathbf{d}$ superimposed experimental spectra (as smoothed contour lines) and the integral of the optical electric field in the SY layer. (Color figure online)

centers of both metallic nanofilms, and the losses of optical waves are small. In the experiments, both metal nanolayers had a thickness of $d_{\mathrm{m}}=18 \mathrm{~nm}$, and from Eq. (1) one obtains $\varrho_{1 / 2}=1.003$ (for $\lambda=575 \mathrm{~nm}$ and $n_{e} \simeq 1$ ). Thus, the experimental value given above agrees well with this value.

\subsection{Dispersion Plots}

Figure 3 demonstrates two-dimensional dispersion plots of the light-emitting structure under study in coordinates $(\varrho$, $\lambda$ ). In Fig. 3a, the magnitude of the optical-field enhancement near the external interface with the air is depicted in color tones with the logarithmic color scale (shown to the right). This field enhancement was calculated for a real structure containing the 27 layers of $\mathrm{SiO}_{2} / \mathrm{Ta}_{2} \mathrm{O}_{5}$ in the $1 \mathrm{D}$ PC. The two black curves are the dispersion curves that were determined for a structure with a semi-infinite 1D PC (see work [24] for more details).

Figure 3a shows that two corresponding modes display anticrossing, and one mode is shifted to the light lineshown as the dashed line of total internal reflection $\rho_{\text {TIR }}$. The error bar represents the imaginary part of the effective RI $\varrho$, which decreases when the LRSP curve approaches the light line at the point $(1.003,575 \mathrm{~nm})$.

The reciprocity theorem $[33,34]$ was used to simulate the experimentally recorded dispersion of electroluminescence, which is shown in Fig. 3b (and which was certainly recorded in the far-field zone). In the present case, it means that the optical electric field, created in the far-field zone by dipoles located in the SY layer-with coordinates from 0 to $\mathrm{d}$, as in Figs. $1 \mathrm{~b}$ and 4 -is the same as the electric field of dipoles from the far-field zone (i.e., 


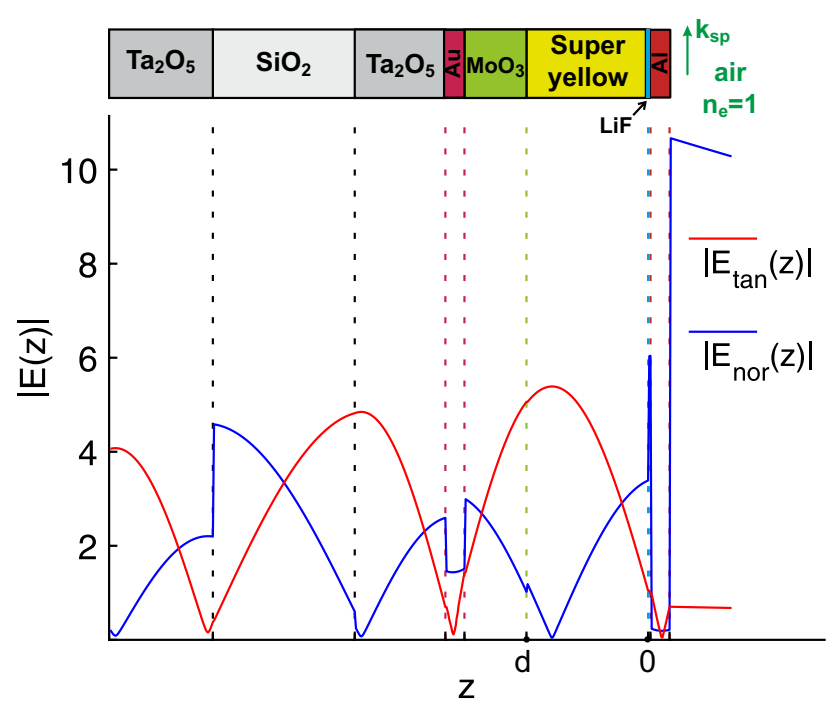

Fig. 4 Spatial distribution of the optical-field components in the outer layers of the structure, at $\lambda=575 \mathrm{~nm}$ and $\varrho=1.003$

from plane optical waves) created in the layer $[0 d]$. The integral of intensity distribution in the SY layer (when the structure is excited by plane waves at different angles of incidence $Q$ and wavelengths $\lambda$ ) is shown in Fig. 3c in color tones. Figure $3 \mathrm{~d}$ depicts the superposition of the calculated integral of the optical electric field in the Super Yellow (SY) layer, from Fig. 3c, and the experimental spectra, from Fig. 3b (depicted as a smoothed contour plot), with a good agreement between these two figures.

\subsection{Field Profiles}

The electric-field profiles in the outer layers of the structure under study are shown in Fig. 4. These profiles were calculated for LRSP excitation at $\lambda=575 \mathrm{~nm}$ and $\varrho=1.003$. The tangential component of the optical field is indeed equal to zero at the centers of both metal nanolayers (as it should be at $\varrho_{1 / 2}$ ), and the total optical field in the metal reaches a minimum with these parameters. This approach ensures minimal energy loss in the metal and maximum propagation length. Notably, in contrast to hybrid dielectric-loaded plasmonic waveguides [35, 36], which are created to achieve the best trade-off between the mode confinement and propagation loss, our multilayer system is designed to achieve minimal propagation loss (even at the cost of the mode confinement). More information on designing LRSP-supporting structures with two metal layers can be found elsewhere [24].

\section{Conclusions}

A current-driven source of LRSPs in a multilayer structure containing an OLED, bounded by two metal nanolayers, on top of a one-dimensional photonic crystal was developed. Electroluminescence spectra were recorded at various decoupling angles, which provided the dispersion profile of the system under study. The LRSP resonance manifested itself at $\lambda=575 \mathrm{~nm}$, near the light line, as expected from the structure design.

The obvious next step is an attempt to achieve LRSP amplification and lasing in the structure with two metal nanolayers. Lasing in the classical symmetric LRSP-supporting structure with one metal nanolayer has been reported for InGaAs quantum-well gain media [37]. However, this was regarding a device with optical pumping, as all SPASERs are today, to the best of the authors' knowledge (for review of amplification and lasing in LRSP-supporting systems, see [38]). It is hoped that the presented approach will lead to electrical pumping in future SPASERs. Lasing in organic media is itself a challenge; nevertheless, there has been a recent report on current-injection lasing from an organic semiconductor [39], indicating that even an organic current-injection SPASER can be potentially possible.

Acknowledgements This work was supported by the Swiss National Science Foundation (Grant 200021_162767).

Open Access This article is licensed under a Creative Commons Attribution 4.0 International License, which permits use, sharing, adaptation, distribution and reproduction in any medium or format, as long as you give appropriate credit to the original author(s) and the source, provide a link to the Creative Commons licence, and indicate if changes were made. The images or other third party material in this article are included in the article's Creative Commons licence, unless indicated otherwise in a credit line to the material. If material is not included in the article's Creative Commons licence and your intended use is not permitted by statutory regulation or exceeds the permitted use, you will need to obtain permission directly from the copyright holder. To view a copy of this licence, visit http://creativecommons.org/licenses/by/4.0/.

\section{References}

1. D.J. Bergman, M.I. Stockman, Surface plasmon amplification by stimulated emission of radiation: quantum generation of coherent surface plasmons in nanosystems. Phys. Rev. Lett. 90, 027402 (2003). https://doi.org/10.1103/PhysRevLet t.90.027402 
2. M.A. Noginov, G. Zhu, A.M. Belgrave, R. Bakker, V.M. Shalaev et al., Demonstration of a spaser-based nanolaser. Nature 460, 1110-1112 (2009). https://doi.org/10.1038/nature08318

3. R.F. Oulton, V.J. Sorger, T. Zentgraf, R.-M. Ma, C. Gladden, L. Dai, G. Bartal, X. Zhang, Plasmon lasers at deep subwavelength scale. Nature 461, 629-632 (2009). https://doi. org/10.1038/nature08364

4. Y.-J. Lu, J. Kim, H.-Y. Chen, C. Wu, N. Dabidian et al., Plasmonic nanolaser using epitaxially grown silver film. Science 337, 450-453 (2012). https://doi.org/10.1126/science.12235 04

5. W. Zhou, M. Dridi, J.Y. Suh, C.H. Kim, D.T. Co, M.R. Wasielewski, G.C. Schatz, T.W. Odom, Lasing action in strongly coupled plasmonic nanocavity arrays. Nat. Nanotechnol. 8, 506-511 (2013). https://doi.org/10.1038/nnano.2013.99

6. F. van Beijnum, P.J. van Veldhoven, E.J. Geluk, M.J.A. de Dood, G.W. 't Hooft, M.P. van Exter, Surface plasmon lasing observed in metal hole arrays. Phys. Rev. Lett. 110, 206802 (2013). https://doi.org/10.1103/PhysRevLett.110.206802

7. C.-J. Lee, H. Yeh, F. Cheng, P.-H. Su, T.-H. Her et al., Lowthreshold plasmonic lasers on a single-crystalline epitaxial silver platform at telecom wavelength. ACS Photon. 4, 14311439 (2017). https://doi.org/10.1021/acsphotonics.7b00184

8. J. Lu, M. Jiang, M. Wei, C. Xu, S. Wang, Z. Zhu, F. Qin, Z. Shi, C. Pan, Plasmon-induced accelerated exciton recombination dynamics in $\mathrm{ZnO} / \mathrm{Ag}$ hybrid nanolasers. ACS Photon. 4, 2419-2424 (2017). https://doi.org/10.1021/acsphotoni cs. $7 \mathrm{~b} 00476$

9. S. Pourjamal, T.K. Hakala, M. Nečada, F. Freire-Fernández, M. Kataja et al., Lasing in Ni nanodisk arrays. ACS Nano 13, 5686-5692 (2019). https://doi.org/10.1021/acsnano.9b01006

10. D. Wang, W. Wang, M.P. Knudson, G.C. Schatz, T.W. Odom, Structural engineering in plasmon nanolasers. Chem. Rev. 118, 2865-2881 (2018). https://doi.org/10.1021/acs.chemr ev. $7 \mathrm{~b} 00424$

11. D. Sarid, Long-range surface-plasma waves on very thin metal films. Phys. Rev. Lett. 47, 1927-1930 (1981). https:// doi.org/10.1103/PhysRevLett.47.1927

12. A.E. Craig, G.A. Olson, D. Sarid, Experimental observation of the long-range surface-plasmon polariton. Opt. Lett. 8, 380 (1983). https://doi.org/10.1364/OL.8.000380

13. P. Berini, Highlighting recent progress in long-range surface plasmon polaritons: guest editorial. Adv. Opt. Photon. 11, ED19 (2019). https://doi.org/10.1364/AOP.11.00ED19

14. V.N. Konopsky, E.V. Alieva, Long-range propagation of plasmon polaritons in a thin metal film on a one-dimensional photonic crystal surface. Phys. Rev. Lett. 97, 253904 (2006). https ://doi.org/10.1103/PhysRevLett.97.253904

15. V.N. Konopsky, E.V. Alieva, Long-range plasmons in lossy metal films on photonic crystal surfaces. Opt. Lett. 34, 479 (2009). https://doi.org/10.1364/OL.34.000479

16. V.N. Konopsky, D.V. Basmanov, E.V. Alieva, S.K. Sekatskii, G. Dietler, Size-dependent hydrogen uptake behavior of pd nanoparticles revealed by photonic crystal surface waves. Appl. Phys. Lett. 100, 083108 (2012). https://doi. org/10.1063/1.3690085

17. S.M. Hamidi, R. Ramezani, A. Bananej, Hydrogen gas sensor based on long-range surface plasmons in lossy palladium film placed on photonic crystal stack. Opt. Mater. 53, 201-208 (2016). https://doi.org/10.1016/j.optmat.2016.01.050

18. E.V. Alieva, V.N. Konopsky, D.V. Basmanov, S.K. Sekatskii, G. Dietler, Blue surface plasmon propagation along thin gold film-gas interface and its use for sensitive nitrogen dioxide detection. Opt. Commun. 309, 148-152 (2013). https://doi. org/10.1016/j.optcom.2013.05.058

19. D.O. Ignatyeva, G.A. Knyazev, P.O. Kapralov, G. Dietler, S.K. Sekatskii, V.I. Belotelov, Magneto-optical plasmonic heterostructure with ultranarrow resonance for sensing applications. Sci. Rep. 6, 28077 (2016). https://doi.org/10.1038/srep28077

20. V.N. Konopsky, E.V. Alieva, S. Yu Alyatkin, A.A. Melnikov, S.V. Chekalin, V.M. Agranovich, Phase-matched third-harmonic generation via doubly resonant optical surface modes in 1d photonic crystals. Light Sci. Appl. 5, e16168 (2016). https ://doi.org/10.1038/lsa.2016.168

21. N.R. Fong, M. Menotti, E. Lisicka-Skrzek, H. Northfield, A. Olivieri et al., Bloch long-range surface plasmon polaritons on metal stripe waveguides on a multilayer substrate. ACS Photon. 4, 593-599 (2017). https://doi.org/10.1021/acsphotoni cs.6b00930

22. V. Konopsky, Long-range surface plasmon amplification with current injection on a one-dimensional photonic crystal surface. Opt. Lett. 40, 2261 (2015). https://doi.org/10.1364/ OL.40.002261

23. I. Degli-Eredi, J.E. Sipe, N. Vermeulen, TE-polarized graphene modes sustained by photonic crystal structures. Opt. Lett. 40, 2076 (2015). https://doi.org/10.1364/OL.40.00207 6

24. V.N. Konopsky, Long-range surface plasmons on duplex metal nanolayers. Photonics Nanostructures - Fundam. Appl. (in press) (2020)

25. P.B. Johnson, R.W. Christy, Optical constants of the noble metals. Phys. Rev. B. 6, 4370-4379 (1972). https://doi. org/10.1103/PhysRevB.6.4370

26. A.D. Rakić, A.B. Djurišić, J.M. Elazar, M.L. Majewski, Optical properties of metallic films for vertical-cavity optoelectronic devices. Appl. Opt. 37, 5271 (1998). https://doi. org/10.1364/AO.37.005271

27. A.D. Rakić, Algorithm for the determination of intrinsic optical constants of metal films: application to aluminum. Appl. Opt. 34, 4755 (1995). https://doi.org/10.1364/AO.34.004755

28. J.H. Youn, S.J. Baek, H.P. Kim, D.H. Nam, Y. Lee, J.G. Lee, J. Jang, Improving the lifetime of a polymer light-emitting diode by introducing solution processed tungsten-oxide. J. Mater. Chem. C 1, 3250 (2013). https://doi.org/10.1039/c3tc00855j

29. N.A. Talik, K.L. Woon, B.K. Yap, W.S. Wong, T.J. Whitcher et al., Highly efficient processable molybdenum trioxide as a hole blocking interlayer for super-yellow organic light emitting diode. J. Phys. D-Appl. Phys. 49, 395105 (2016). https://doi. org/10.1088/0022-3727/49/39/395105 
30. J.C. Bernède, L. Cattin, S.O. Djobo, M. Morsli, S.R.B. Kanth et al., Influence of the highest occupied molecular orbital energy level of the donor material on the effectiveness of the anode buffer layer in organic solar cells. Phys. Status Solidi 208, 1989-1994 (2011). https://doi.org/10.1002/pssa.20112 7047

31. V.N. Konopsky, Plasmon-polariton waves in nanofilms on onedimensional photonic crystal surfaces. New J. Phys. 12, 093006 (2010). https://doi.org/10.1088/1367-2630/12/9/093006

32. F. Yang, J.R. Sambles, G.W. Bradberry, Long-range surface modes supported by thin films. Phys. Rev. B 44, 5855-5872 (1991). https://doi.org/10.1103/PhysRevB.44.5855

33. H. Yang, N. Alexopoulos, Gain enhancement methods for printed circuit antennas through multiple superstrates. IEEE Trans. Antennas Propag. 35, 860-863 (1987). https://doi. org/10.1109/TAP.1987.1144186

34. X.H. Wu, A.A. Kishk, A.W. Glisson, A transmission line method to compute the far-field radiation of arbitrarily directed hertzian dipoles in a multilayer dielectric structure: theory and applications. IEEE Trans. Antennas Propag. 54, 2731-2741 (2006). https://doi.org/10.1109/TAP.2006.882164
35. H.S. Chu, P. Bai, E.P. Li, W.R.J. Hoefer, Hybrid dielectricloaded plasmonic waveguide-based power splitter and ring resonator: compact size and high optical performance for nanophotonic circuits. Plasmonics 6, 591-597 (2011). https ://doi.org/10.1007/s11468-011-9239-y

36. D. Dai, H. Wu, W. Zhang, Utilization of field enhancement in plasmonic waveguides for subwavelength light-guiding, polarization handling, heating, and optical sensing. Materials 8, 6772-6791 (2015). https://doi.org/10.3390/ma8105341

37. R.A. Flynn, C.S. Kim, I. Vurgaftman, M. Kim, J.R. Meyer et al., A room-temperature semiconductor spaser operating near $15 \mu \mathrm{m}$. Opt. Express 19, 8954 (2011). https://doi. org/10.1364/OE.19.008954

38. P. Berini, I. De Leon, Surface plasmon-polariton amplifiers and lasers. Nat. Photon. 6, 16-24 (2012). https://doi. org/10.1038/nphoton.2011.285

39. A.S.D. Sandanayaka, T. Matsushima, F. Bencheikh, S. Terakawa, W.J. Potscavage et al., Indication of current-injection lasing from an organic semiconductor. Appl. Phys. Express 12, 061010 (2019). https://doi.org/10.7567/1882-0786/ab1b90 